\title{
DEA for soft robotics: 1-gram actuator picks up a 60-gram egg
}

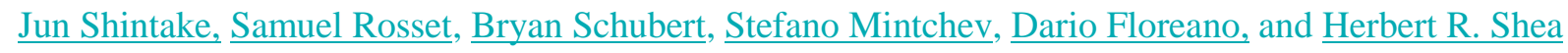

"DEA for soft robotics: 1-gram actuator picks up a 60-gram egg”, Proc. SPIE 9430, Electroactive Polymer Actuators and Devices (EAPAD) 2015, 94301S (April 1, 2015); doi:10.1117/12.2084043

Copyright 2015 Society of Photo-Optical Instrumentation Engineers. One print or electronic copy may be made for personal use only. Systematic electronic or print reproduction and distribution, duplication of any material in this paper for a fee or for commercial purposes, or modification of the content of the paper are prohibited.

http://dx.doi.org/10.1117/12.2084043 


\title{
DEA for soft robotics: 1-gram actuator picks up a 60-gram egg
}

\author{
Jun Shintake*a,b, Samuel Rosset ${ }^{\mathrm{a}}$, Bryan Schubert ${ }^{\mathrm{b}}$, Stefano Mintchev $^{\mathrm{b}}$, Dario Floreano ${ }^{\mathrm{b}}$, \\ Herbert Shea $^{\text {a }}$ \\ ${ }^{a}$ Microsystems for Space Technologies Laboratory, Ecole Polytechnique Fédérale de \\ Lausanne, Neuchâtel, Switzerland \\ ${ }^{\text {b}}$ Laboratory of Intelligent Systems, Ecole Polytechnique Fédérale de \\ Lausanne, Lausanne, Switzerland
}

\begin{abstract}
We introduce a soft actuator for grippers using DEA capable of bending actuation. The actuator is also able to generate the electro-adhesion by the fringe field formed at the edges of the electrodes. The adhesion improves the holding force and ensures the conformation of the structure to the object. After the characterization of the actuator, we develop a 2 finger soft gripper capable of holding various objects. The gripper has a mass of around $1 \mathrm{~g}$, and consists of a few cm long actuation parts, realizing simple open-close movement. The compliance of the gripper leads to conformation of the structure against the object surface, which is proven by successful handling of objects with different geometries such as a toothbrush, a flat paper, and a ping pong ball. The effect of the electro-adhesion is visible when the paper is held with its flat shape meaning that an adhesion force against gravity exists. Also, by the fact that the conformed structure increases the contact area, the holding force is improved while avoiding damaging the object, which is highlighted by the ability to hold a raw egg weighing around $60 \mathrm{~g}$. This soft gripper, combining both actuation and electro-adhesion, illustrates the potential use of DEA for soft robotics.
\end{abstract}

Keywords: Soft robotics, gripper, bending actuator, electro-adhesion

\section{INTRODUCTION}

Soft robotics is a rising research field where robots are composed of compliant materials in contrast to conventional robots based on rigid materials ${ }^{1,2,3}$. Potential advantages of this approach has been demonstrated in soft grippers where the grasping of a fragile object, and holding of various objects with the conformed structure are achieved by a simple structure and control ${ }^{4,5}$, while showing resistance to mechanical damages ${ }^{6}$. These capabilities are often difficult to achieve by traditional robotic gripper where the system consists of links, motors, and sensors that tend to be complicated or fragile, and in some cases, requiring a sophisticated control.

We are interested in the use of dielectric elastomer actuators (DEAs) $)^{7,8,9}$ for soft grippers, due to their promising features that could be applied to the devices, such as a high compliance ( $\sim 1 \mathrm{MPa}$ of elastic modulus), lightweight, a large actuation stroke (over $1000 \%$ of area expansion ${ }^{10}$ ), fast response $\left(\mathrm{kHz}\right.$ bandwidth ${ }^{11}$ ), and self-sensing capability in closed loop systems ${ }^{12}$. However, a difficulty exists, as DEAs usually provide low stress, which limits the holding force of the gripper. Reported DEA-based bending actuators for a satellite-mounted gripper show a few $\mathrm{mN}$ of gripping force ${ }^{13}$ which is sufficient to grab and hold objects in space, but not on Earth due to gravity. One way to increase the holding force of a DEA-based gripper is to use electro-adhesion ${ }^{14}$. Electro-adhesion is an electrostatic force caused by electric field, commonly used in industry as electrostatic chucks handling wafers and LCD panels, and this technology has been applied to a motor driven gripper ${ }^{15}$.

The objective of this study is to develop a soft gripper using DEA. We introduce a soft bending actuator for gripping applications. Due to the fringe field ${ }^{16}$ formed on the edge of the electrodes, the actuator is able to generate an electroadhesion which can be used to improve the holding force, without need of additional parts. The actuator is characterized in terms of the tip angle as a function of the applied voltage. We then show a 2-finger gripper based on our bending/electro-adhesive actuators and demonstrate the holding of various objects.

*E-mail: jun.shintake@epfl.ch 


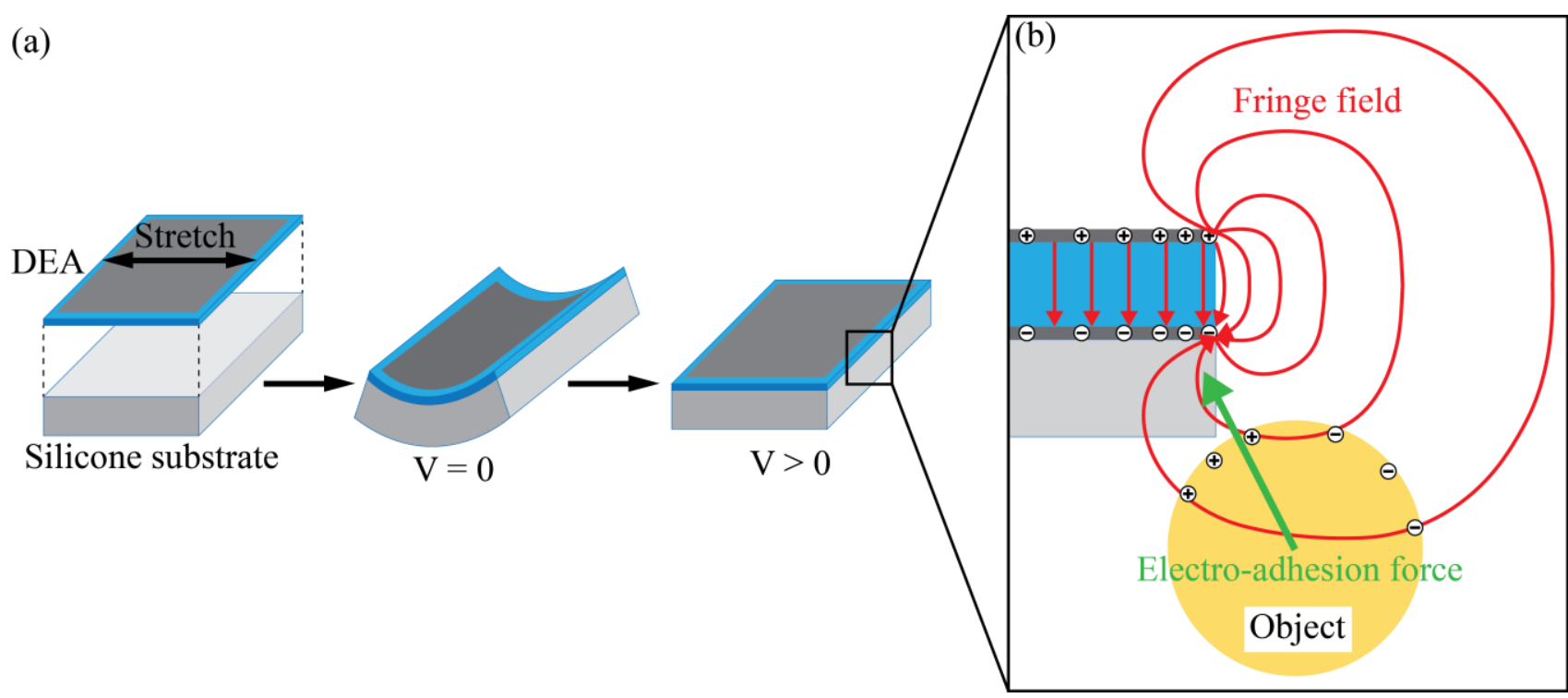

Figure 1. Working principle of the actuator. (a) Bending actuation can be achieved with a pre-stretched DEA bonded onto a silicone substrate. (b) The fringe field formed on the edge of the electrodes generates an electro-adhesion force to the object near the structure.

\section{ACTUATOR CONCEPT}

The actuator is composed of a pre-stretched DEA bonded to a silicone substrate, resulting in a bending shape at no applied voltage, where the strain energy of the DEA and the bending energy of the substrate are minimized, as shown in Figure 1(a). When a voltage is applied, the electrostatic energy in the DEA shifts the energy balance towards the state where the substrate is flat. At the same time, the electric field of the DEA is formed inside of the structure and the outside, as shown in Figure 1(b). The field inside contributes to generate the Maxwell pressure resulting in the actuation of the DEA. On the other hand, the field outside, so-called the fringe field is generated along the edge of the electrodes. The fringe field generates an electro-adhesion on close objects. As the result, the device combines actuation and electroadhesion in a single, simple structure. For grippers, the compliance of the actuator leads to the conformation of the structure to the object resulting in increased contact area, which improves the holding force in the shear direction. The large contact area also provides distributed force on the interface for avoiding damaging the object. The electro-adhesion ensures the conformation of the structure therefore reinforces the holding force in the shear direction by adding the normal force.

The configuration of the actuator is similar to the dielectric elastomer minimum energy structures (DEMESs) proposed by Kofod et al. ${ }^{17}$, however, different features can be obtained. (1) Compared to DEMES, the entire structure is compliant, which leads to a better conformation of the structure to the object. (2) The absence of a central hole also makes the fabrication process easier and the actuator more robust, as the DEA membrane is protected by the soft substrate.

\section{FABRICATION AND CHARACTERIZATION OF THE ACTUATOR}

We fabricated and characterized the actuator shown in Figure 2(a). The device has a rectangular geometry (32 mm length and $12 \mathrm{~mm}$ width). We used the silicone elastomers CF19-2186 (NuSil Technology) for the DEA membrane, and LSR4305 (Bluestar Silicones) for the substrate. After the casting and the curing at temperature of $80{ }^{\circ} \mathrm{C}$, the DEA membrane (thickness of $\sim 50 \mu \mathrm{m}$ ) is pre-stretched uniaxially with a ratio of 1.25 , and the electrodes made with a soft silicone and carbon black were applied on to the membrane surfaces using a stamping method. Subsequently, the DEA was attached onto the casted silicone substrate (thickness $2 \mathrm{~mm}$ ) using oxygen plasma surface activation (Zepto plasma 


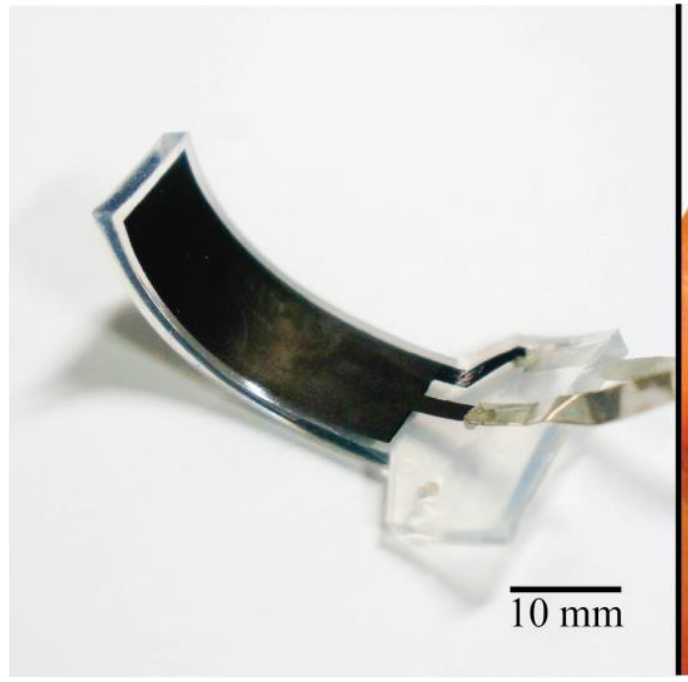

(a)

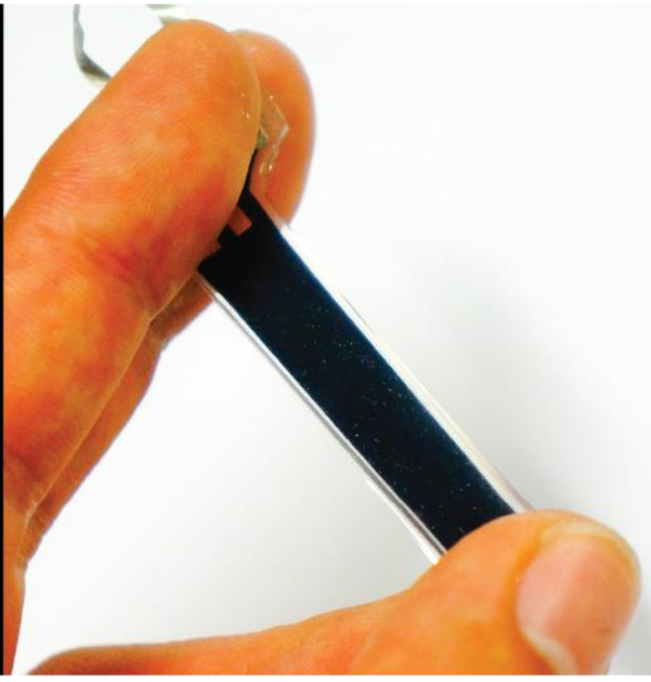

(b)

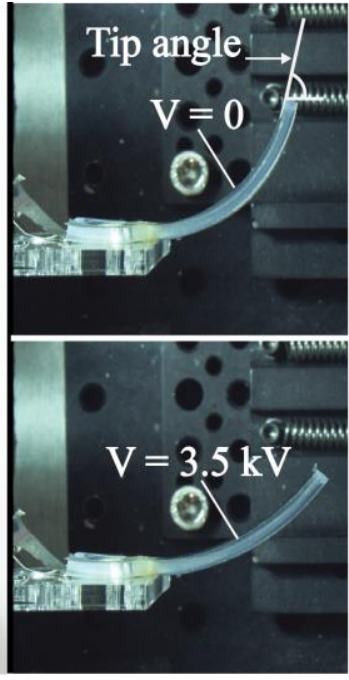

(c)

Figure 2. (a) Fabricated actuator. (b) The actuator can be stretched thanks to its compliance. (c) Actuation of the device.

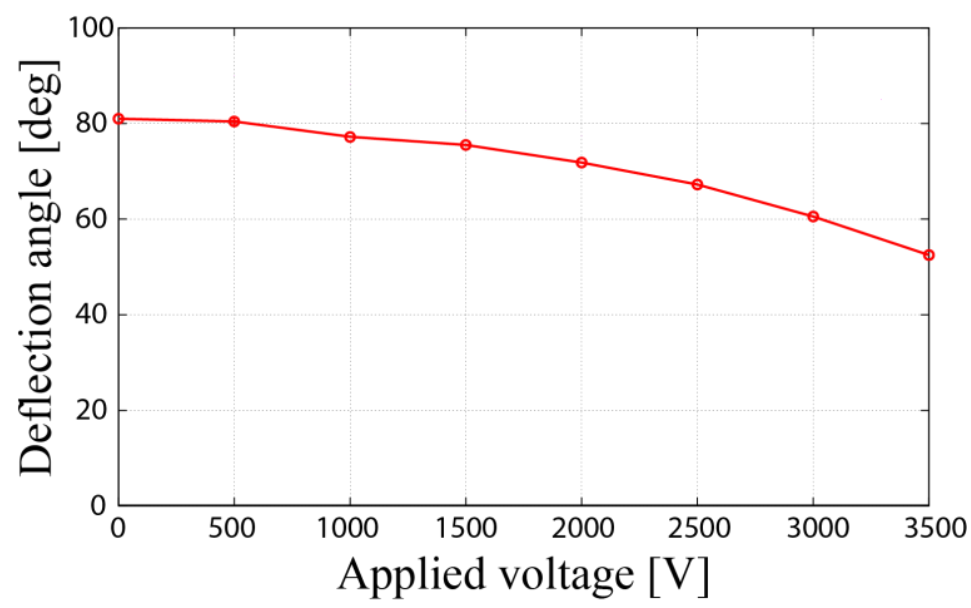

Figure 3. Tip angle of the actuators as a function of the applied voltage. The actuator shows the controllable actuation range of $28.5^{\circ}$ at $3.5 \mathrm{kV}$.

system, diener). We used a film coater (ZAA2300, Zehntner) and a film applicator (ZUA2000, Zehntner) for the membrane casting. The entire structure of the actuators is soft thanks to the use of the silicones (Figure 2(b)).

Figure 2(c) shows the movement of the actuator for a given applied voltage. The device performs the actuation towards the flat state. Figure 3 shows a result on characterizing the tip angle of the actuator as a function of the applied voltage. Both of the actuators exhibit controllable bending angle. The maximum actuation angle was obtained as $28.5^{\circ}$ at $3.5 \mathrm{kV}$.

\section{SOFT GRIPPER USING THE ACTUATOR}

We developed a soft gripper to see the usefulness of the actuator. The gripper shown in Figure 4(a) consists of 2 actuators. Each actuator has length of $42.5 \mathrm{~mm}$ with a triangular shape. As shown in Figure (b), the movement of the gripper is a simple open-close behavior, where the silicone substrate of the actuator has the contact against the object. The fringe field generated from the DEA penetrates the substrate, and causes the electro-adhesion in the interface 


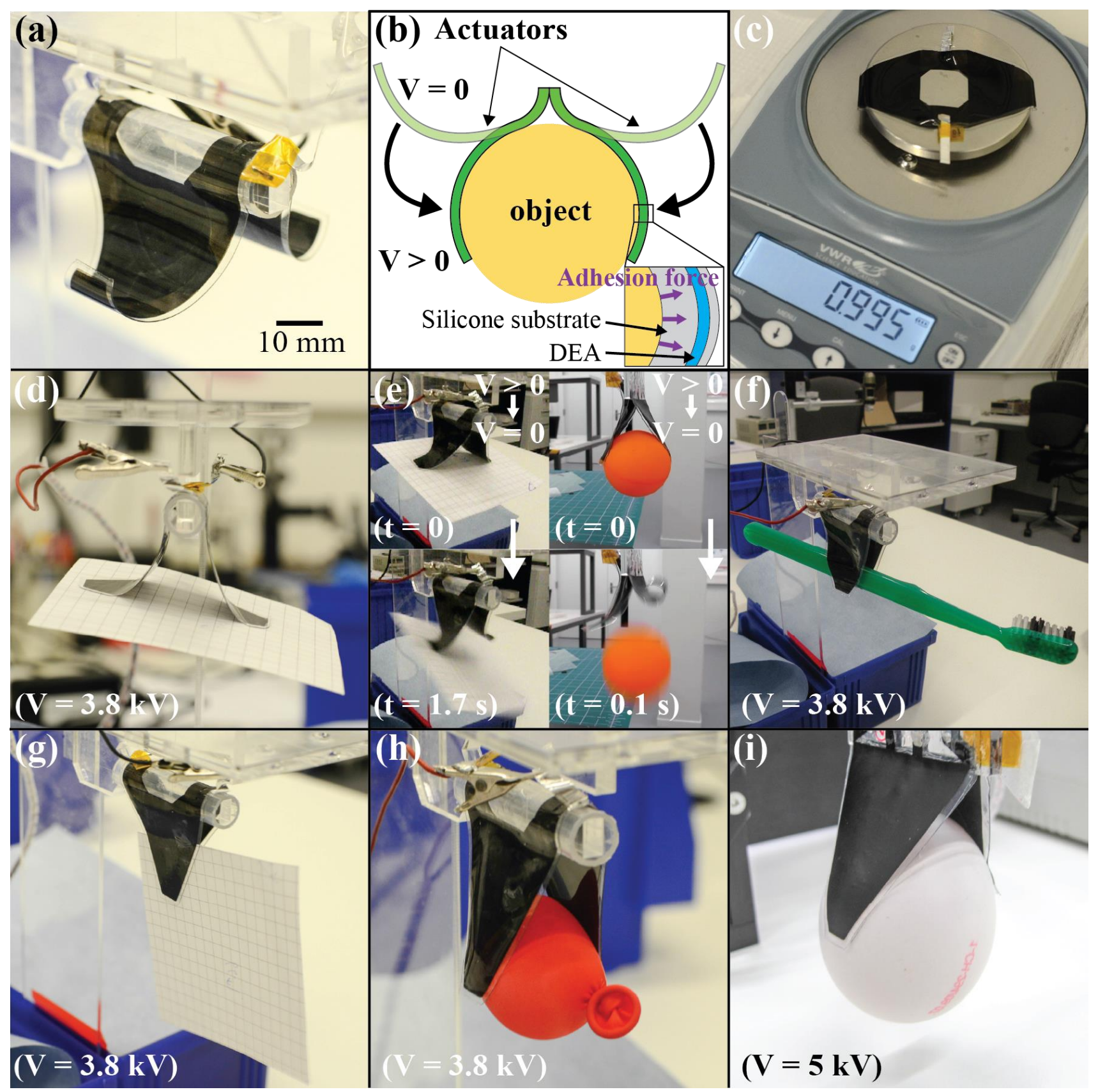

Figure 4. (a) Developed soft gripper. (b) The actuators realizes a simple open-close movement. (c) Thanks to the simple structure and lightweight materials, the entire mass of the device is around $1 \mathrm{~g}$. (d) A flat paper is successfully held thanks to the electro-adhesion. (e) Releasing of objects is possible by simply cutting off the voltage. Holding of objects with different geometry has been demonstrated for: (f) a tooth brush, (g) pinching of a thin object (a flat paper), (h) grasping of a soft object (a water balloon), and (i) a raw egg.

between the substrate and the object surface. The intensity of the adhesion depends on the distance between the DEA and the object surface. This means the thinner substrate, the larger the electro-adhesion force. For this purpose, we used a stiffer silicone (Sylgard 184, Dow Corning) for the substrate to decrease its thickness $(\sim 150 \mu \mathrm{m})$, while keeping the same stiffness for the DEA. Sylgard 184 has an elastic modulus around $1.9 \mathrm{MPa}$ at curing temperature of $80{ }^{\circ} \mathrm{C}^{18}$. Additionally, a cover layer made of the same silicone (thickness $\sim 50 \mu \mathrm{m}$ ) is put on the top of the DEA to adjust the initial angle of the 
actuator. For the DEA, a membrane made of CF19-2816 (thickness $\sim 60 \mu \mathrm{m}$ ) was used with uniaxial pre-stretch of 1.2. Due to the simple structure, the mass of the gripper is around $1 \mathrm{~g}$ including conductive tapes for the electric contact (Figure 4(c)). We tested the capability of the gripper in terms of the holding of the object. For this purpose, the device was fixed on a setup using plastic parts, and different objects were approached from the bottom to emulate pick-andplace movement, which is a common behavior of grasping devices. Figure 4(d) shows a thin flat object (a paper) held with its flat shape without grasping but adhering, where the structure of the gripper is conformed to the object horizontally, exhibiting an electro-adhesion force in vertical direction against gravity. This result shows a potential functionality of the gripper allowing handling of flat objects.

Releasing of the object is possible by simply cutting off the voltage (e.g., Figure 4 (e), with a paper and a ping pong ball), where the use of the stiffer silicone (Sylgard 184) minimizes the stiction of the material and therefore enhances the separation from the object surface. A fast releasing time for the ping pong ball was observed, and it is due to the fast response of the DEA. The releasing time was slower for the paper, this may have resulted from the remaining charges on the electrodes and the low mass of the object. The gripper performed successful holding for various objects including a toothbrush, a vertical paper, a water balloon as a soft object, and a raw egg as a fragile object (Figure (f-i)). Thanks to the compliance, the structure of the gripper is conformed along the geometry of the object. The conformed structure increases contact area, therefore the holding force, while the electro-adhesion assists them. These effects are highlighted in the holding of the raw egg which is fragile and heavy object $(60 \mathrm{~g})$. The holding behaviors shown in Figure 4 are achieved with a single voltage input (on and off), i.e., simplified control.

\section{CONCLUSION}

We proposed, fabricated, and characterized a bending actuator using DEA for soft gripper. The device is able not only of actuation but also to provide an electro-adhesion force, which improves the holding force. The characterization result of the actuator presented the voltage-controllable bending angle. The developed gripper proved the concept by successfully holding various objects, while exhibiting the effect of the electro-adhesion. Our future work includes characterization of the electro-adhesion generated from DEAs, modelling of both the electro-adhesion and the actuation, and further investigation on the grasping capability of the gripper. The demonstration of a working soft gripper combining Maxwellpressure actuation with electro-adhesion, and the fact that the behavior of the device can be achieved with a simple structure and control illustrate the potential use of DEA for soft robotics.

\section{ACKNOWLEDGMENTS}

We gratefully acknowledge financial supports from the Swiss National Center for Competence in Research (NCCR) Robotics (Project No. 1.2 Sensorymotor tissues), and from the Swiss National Science Foundation, grant \#200020153122 .

\section{REFERENCES}

1. D. Trivedi, C. D. Rahn, W. M. Kier, and I. D. Walker, "Soft robotics: Biological inspiration, state of the art, and future research," Applied Bionics and Biomechanics 5(3), pp. 99-117, 2008.

2. R. Pfeifer, M. Lungarella, and F. Iida, "The Challenges Ahead for Bio-Inspired 'Soft' Robotics," Communications of the ACM 55(11), pp. 6-87, 2012.

3. C. Majidi, "Soft Robotics: A Perspective - Current Trends and Prospects for the Future," Soft Robotics, 1(1), pp. 5-11, 2014.

4. F. Ilievski, A. D. Mazzeo, R. F. Shepherd, X. Chen, and G. M. Whitesides, "Soft Robotics for Chemists," Angewandte Chemie International Edition, 50(8), pp. 1890-1895, 2011.

5. E. Brown, N. Rodenberg, J. Amend, A. Mozeika, E. S. M. R. Zakin, H. Lipson, and H. M. Jaeger, "Universal robotic gripper based on the jamming of granular material," Proceedings of the National Academy of Sciences, 107(44), pp. 18809-18814, 2010.

6. R. V. Martinez, A. C. Glavan, C. Keplinger, A. I. Oyetibo, and G. M. Whitesides, "Soft Actuators and Robots that Are Resistant to Mechanical Damage," Advanced Functional Materials, 24(20), pp. 3003-3010, 2014.

7. R. E. Pelrine, R. D. Kornbluh, and J. P. Joseph, "Electrostriction of polymer dielectrics with compliant electrodes as a means of actuation," Sensors and Actuators A: Physical 64(1), pp. 77-85, 1998. 
8. I. A. Anderson, T. A. Gisby, T. G. McKay, B. M. O’Brien, and E. P. Calius, "Multi-functional dielectric elastomer artificial muscles for soft and smart machines," Journal of Applied Physics, 112(4), pp. 041101-1041101-20, 2012.

9. S. Rosset, and H. R. Shea. "Flexible and Stretchable Electrodes for Dielectric Elastomer Actuators," Applied Physics A: Materials Science 110(2), pp. 281-307, 2013.

10. T. Li, C. Keplinger, R. Baumgartner, S. Bauer, W. Yang, and Z. Suo, "Giant voltage-induced deformation in dielectric elastomers near the verge of snap-through instability," Journal of the Mechanics and Physics of Solids, 61(2), pp. 611-628, 2013.

11. R. Kornbluh, R. Peirine, Q. Pei, S. Oh, and J. Joseph, "Ultrahigh strain response of field-actuated elastomeric polymers," in Proc. SPIE, San Diego, CA, USA, Mar. 2000, 3987, pp. 51-64.

12. T. A. Gisby, E. P. Calius, S. Xie, and I. A. Anderson, "An adaptive control method for dielectric elastomer devices,” in Proc. SPIE, San Diego, USA, Mar. 2008, 6927, pp. 69271C-1-69271C-8.

13. O. A. Araromi, I. Gavrilovich, J. Shintake, S. Rosset, M. Richard, V. Gass, and H. Shea, "Roll-able Multisegment Dielectric Elastomer Minimum Energy Structures for a Deployable Microsatellite Gripper," IEEE/ASME Transactions on Mechatronics, 20(1), pp. 438-446, 2015.

14. K. Asano, F. Hatakeyama, and K. Yatsuzuka, "Fundamental Study of an Electrostatic Chuck for Silicon Wafer Handling”, IEEE Transactions on Industry Applications, 38(3), pp. 840-845, 2002.

15. Grabitinc.com, 'Products | Grabit Inc', 2015. [Online]. Available: http://grabitinc.com/products/. [Accessed: 26Jan- 2015].

16. J. Vanderlinde, Classical Electromagnetic Theory, Springer, pp. 119-120, 2005.

17. G. Kofod, W. Wirges, M. Paajanen, and S. Bauer, "Energy minimization for self-organized structure formation and actuation," Applied Physics Letters 90(8), p. 081916, 2007.

18. I. D. Johnston, D. K. McCluskey, C. K. L. Tan, and M. C. Tracey, "Mechanical characterization of bulk Sylgard 184 for microfluidics and microengineering," Journal of Micromechanics and Microengineering, 24(3), p. 035017, 2014. 